\title{
Enhancement of irradiation resistance and experimental research of compact electronics for ITER Radial X-ray Camera
}

\author{
Kaiyun CHEN, Hongrui CAO*, Jinlong ZHAO, Xiuli SHENG, Shi LI, Liqun HU \\ Institute of Plasma Physics, Chinese Academy of Sciences, Hefei 230031, China. \\ *Corresponding author: caohr@ipp.ac.cn
}

\begin{abstract}
International Thermonuclear Experimental Reactor (ITER) Radial X-ray Camera (RXC) is installed in the equatorial port \#12 (middle drawer) to measure the poloidal profile of the plasma $x$-ray emission with high spatial and temporal resolution. According to the irradiation test results with Co-60 gamma ray source, Cf-252 neutron source and the result in Deuterium Tritium (DT) accelerator neutron irradiation experiment for RXC electronics before, the weak parts of the circuit have been improved by radiation resistance design such as using radiation-hard power chip and optimizing the Field Programmable Gate Array (FPGA) code. Other factors related to radiation, such as easiness of maintenance, compact electronics structure and remote state monitoring are also considered in the design. The performance of the electronics and chassis has been preliminarily verified in Experimental Advanced Superconducting Tokamak (EAST) experiments showing resistance to interference from discharge environment. Besides, the irradiation resistance of electronics was investigated in high fluence neutron irradiation experiment in fission reactor, where the detector, pre-amplifier, mid-amplifier and cables under test received the accumulated neutron fluence over $9.89 \times 10^{13} \mathrm{n} / \mathrm{cm}^{-2}$ (1MeV equivalent), and the quality of output signal was monitored in real time. It's found after the experiment that the pre-amplifier worked well, while the detector and mid-amplifier were partly damaged.
\end{abstract}

Index Terms - Amplifier, Chassis and shielding, Neutron radiation test, $\mathrm{X}$-ray camera.

\section{INTRODUCTION}

International Thermonuclear Experimental Reactor (ITER) Radial X-ray Camera (RXC) is installed in equatorial \#12 (middle drawer) to measure the poloidal profile of the plasma $\mathrm{x}$-ray emission with high spatial and temporal resolution $[1,2]$. To have a whole view of the plasma and keep the detectors from the neutron flux, this camera will be separated into two individual cameras: internal camera and external camera as shown in Fig.1 [1]. Internal camera will be installed inside the port plug and its detectors will be mounted on upper side and bottom side of DSM (Diagnostic Shielding Module) to have the edge views of the plasma. External camera outside of the port plug will have views to cover the core plasma and the detector arrays will be installed in secondary vacuum chamber. In current stage, the detector and electronics used for internal camera are still under development. As a result, only electronics for external camera will be discussed in detail in this paper, although the electronics for internal camera will be quite similar. According to the previously reported irradiation test results with Co-60 gamma ray source, Cf-252 neutron source and the result in Deuterium - Tritium (DT) accelerator neutron irradiation experiment for RXC electronics [2], the weak parts of the circuit are power chip and FPGA, and have been improved by radiation resistance design which is described in the following sections. Meanwhile, considering the nuclear environment (to reduce worker exposure to radiation) and space limitation in port cell (to allow more space to put nuclear shielding), easiness of maintenance, compact electronics structure and remote state monitoring are designed. In addition, design of magnetic shielding, irradiation experiment in reactor and integration test in EAST tokamak are presented.

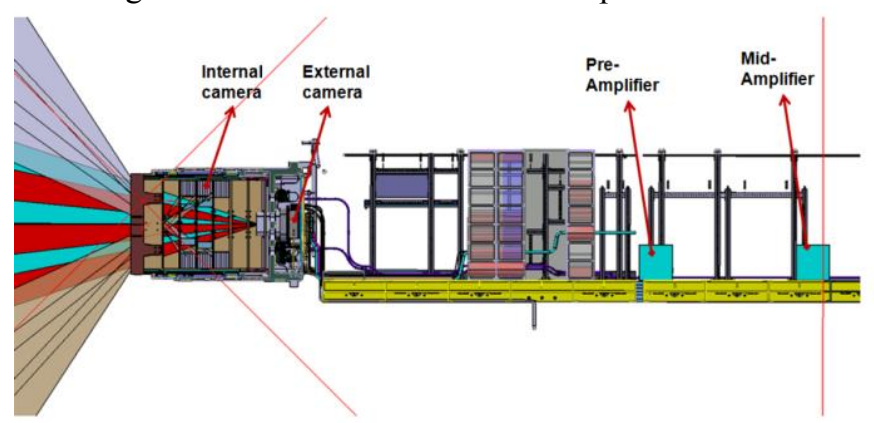

Fig. 1 3D model of the RXC components

\section{ELECTRONICS DESIGN}

As shown in Fig.2, the electronics mainly includes detector, pre-amplifiers, mid-amplifiers and I\&C (Instrument and control). The pre-amplifiers will be placed in the front of port cell 12 close to the bio-shield wall and mid-amplifiers in the 
end of port cell 12 close to the mashing box. Extra-low-voltage power supply will be placed in the corner of diagnostic building. I\&C (including DAQ (Data AcQuistion), fast and slow controllers) will be housed in the cubicle area of diagnostic building.

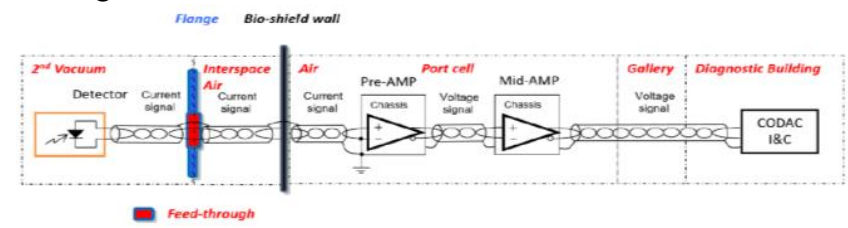

Fig.2 RXC electronics layout

For electronics, the actual measured quantity is chord-integrated soft x-ray intensity with signal current varying from $10 \mathrm{nA}$ to $10 \mathrm{uA}$ and maximum time resolution $20 \mathrm{kHz}$. To enhance the flexibility of electronics to both monitor low performance and high performance plasmas, the gain of pre-amplifier should be fixed at the order of magnitude $1 \times 10^{6} \mathrm{~V} / \mathrm{A}$ and gain of mid-amplifier is designed to be changeable. After a long distance transmission (about 20 meters) from detectors, the current signal from detector is amplified to voltage signal by the trans-impedance pre-amplifier and the output will flow into mid-amplifier which is programmable and is controlled by I\&C. The output signal of mid-amplifier will be sent to the DAQ system and the mid-amplifier can drive long distance cable whose length is up to 100 meters.

Fig. 3 shows the electronics and I\&C architecture. In order to control the gain of mid-amplifier, the radiation resistant fiber to ensure communication is used to connect the I\&C cubicle in the diagnostic building with mid-amplifier. When the gain of mid-amplifier needs to be changed, the fast controller in the I\&C cubicle will send the digital electronical signal to mid-amplifier chassis with communication mode of RS232.

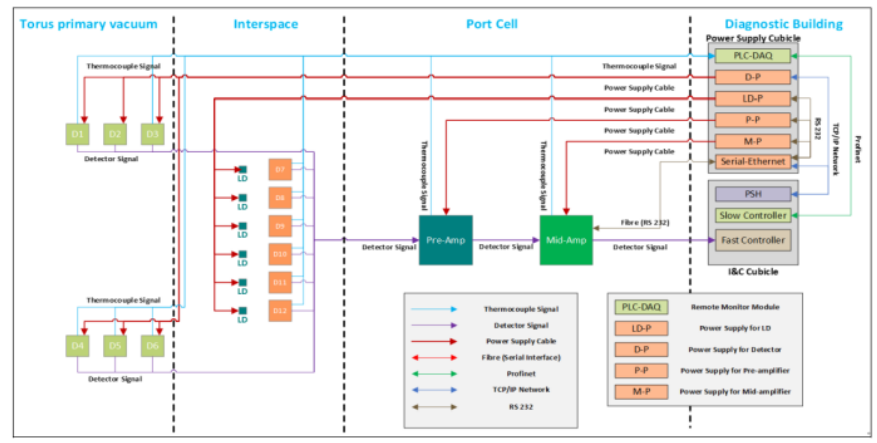

Fig.3 The electronics and I\&C architecture

The performance of pre-amplifier for weak current signal will influence the availability of the whole RXC electronics system. Because of the strong electromagnetic interference in ITER environment, the far-front signal transmission, improvement of signal to noise ratio, resistance to electromagnetic interference and other key technologies need to be investigated deeply.

The principle design of pre-amplifier is shown in Fig. 4 and Fig.5. The pre-amplifier circuit is divided into two stages. The first stage is transimpedance amplification with single-ended input and differential output and mainly uses OPA2140 chip [3]. The second stage is voltage amplification and differential voltage driving which mainly uses THS4131 [4]. In order to improve the nuclear radiation resistant performance of the power supply module, radiation resistant power chips are used in the pre-amplifier design (RHFL4913A and RHFL7913A) [5, $6]$.

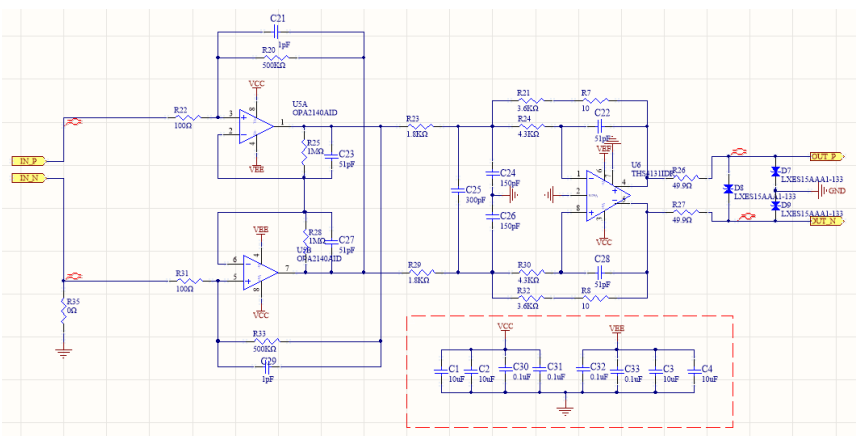

Fig.4 Schematic of differential amplifier part

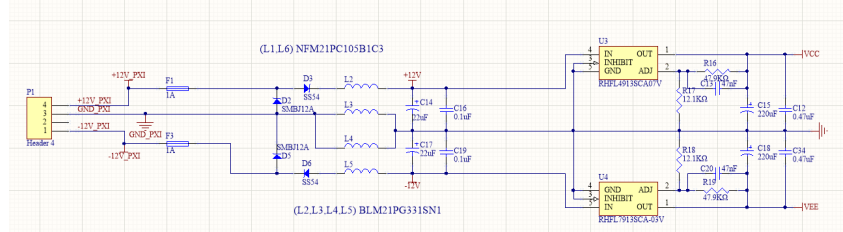

Fig.5 Schematic of power supply part

The principle design of mid-amplifier is shown in Fig.6. One master mid-amplifier and nine slave mid-amplifier boards will be used in the mid-amplifier chassis. The master one includes five parts: 16-channel PGA (programmable gain amplifier), fiber-to-RS232 converting module, FPGA, self-test function part and analog regulated power module. In these five parts, FPGA is a core processing module which can receive gain adjustment command from fiber-to-RS232 module which receive the optical control signal from fast controller in I\&C cubicle and control 16-channel PGA to change the amplification. FPGA can also give a monitoring signal such as operating status to I\&C for checking. The third function of FPGA is to control the switch for mid-amplifier self-test. Self-test module includes 16 switches and a self-test signal generator which is a reference voltage source. When system operates normally, FPGA controls the switch to connect the original signal from pre-amplifier to the input end of PGA in mid-amplifier. If self-test of mid-amplifier is required, FPGA will change the switch to connect the self-test signal generator to the input end of PGA in mid-amplifier. For final design and electronic test, 10M08SAU169C8 FPGA is used from company of Intel [7]. 


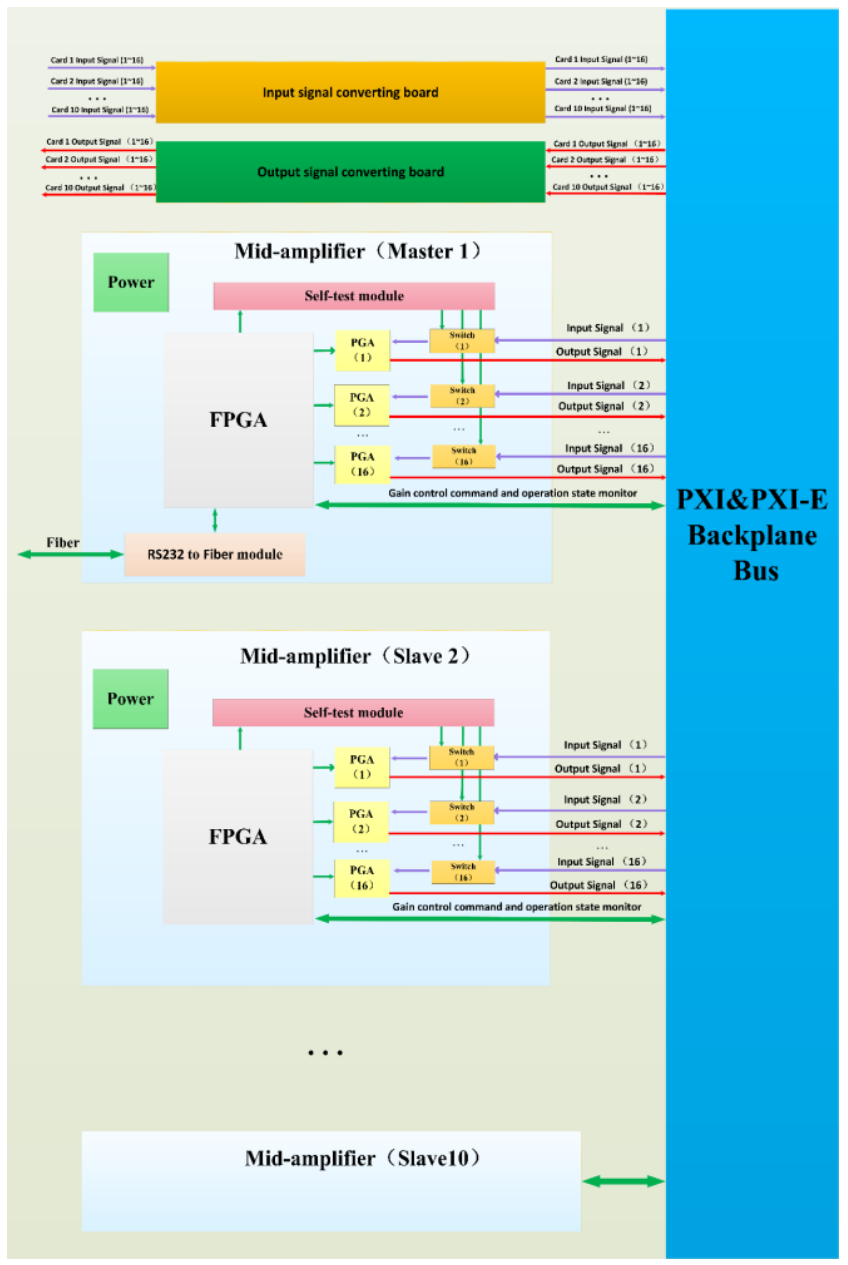

Fig.6 Principle structure of mid-amplifier

In PCB design, pre-amplifier and mid-amplifier are based on PXIE compact architecture as shown in Fig.7 and Fig.8. The multi-channel consistency, heat dissipation and crosstalk have been specially considered in PCB design.

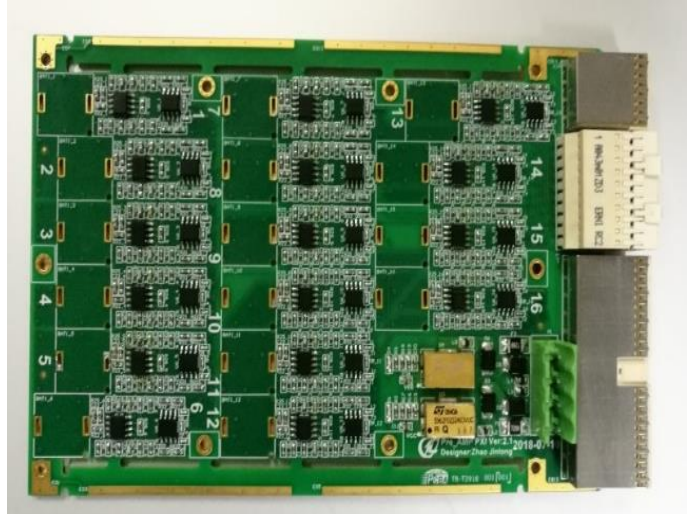

Fig.7 Prototype of pre-amplifier

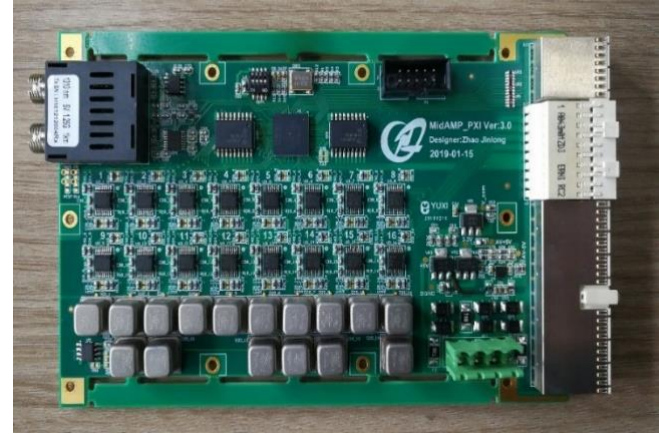

Fig.8 Prototype of mid-amplifier

The state machine is used in the design to remotely control and monitor the working state of mid-amplifier. If a problem is found in mid-amplifier, the mid-amplifier can be restarted by soft reset or hard reset to improve the availability. These designs will reduce human in-situ operations in port cell and consequently occupational exposure. As shown in Fig.9, the state machine has five states: initialization, configuration, self-test, running, and error.

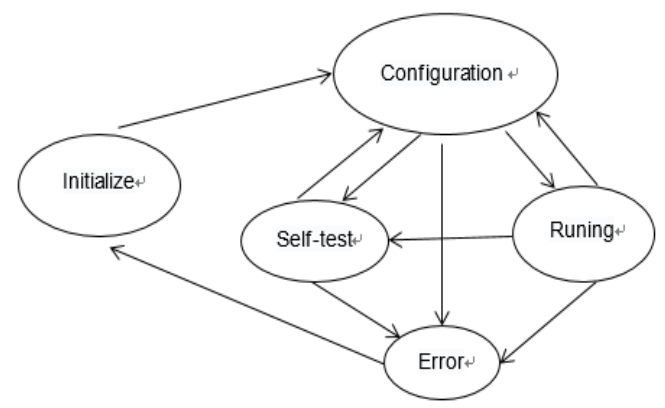

Fig.9 The flow chart of state for FPGA code of mid-amplifier

After the mid-amplifier cards are powered on, the state machine firstly comes to the initialization state, during which all the registers in FPGA are reset, the default gain configuration are restored, the RS232 communication from master mid-amplifier to I\&C system are set up, and the communication from master mid-amplifier to the slave mid-amplifier are established. If the initialization state has been prepared, the state machine jumps to the configuration state automatically. In the configuration state, I\&C system will send the gain configuration command to master mid-amplifier, and master mid-amplifier will send the command to the other slave mid-amplifier. When the configuration is successful done, the mid-amplifier will send back the information showing configuration is ok. And then I\&C will send the command to control the state machine to jump to the self-test state or the running state. In the running state, I\&C system can monitor the gain. If the gain of mid-amplifier need change, I\&C system will send the command to control the state machine to come back to configuration state. In self-test state, I\&C system can control the mid-amplifier to use the internal reference signal source in mid-amplifier circuit board to test signal amplification of the mid-amplifier. When an error occurs in running state or self-teat state, the state machine will come to error state and 
send the information of error to I\&C system. When I\&C system receives the information of error, it will send the command to control the state machine to change to the initialization state.

Soft and hard reset of the mid-amplifier FPGA system is realized in the design. If there is any error of FPGA generated by SEE (Single Event Effect) or other reasons, I\&C control can select soft or hard reset of FPGA. If the hardware reset is selected, I\&C will send a shutdown command to turn off the ELV (Extremely Low Voltage) power in power supply cubicle. After stopping for a few seconds, he turn-on command is sent. The mid-amplifier will restart, and the FPGA will automatically jump to the initialization state, and perform the initialized work. If choosing software restart, I\&C will directly send restart command to the FPGA in mid-amplifier, and the FPGA will jump back to initialize the state and restart the process.

\section{CHASSIS AND SHIELDING DESIGN}

The pre-amplifiers and mid-amplifiers are housed in chassis. The chassis as shown in Fig.10, which is designed based on PXI/PXIE mixed bus architecture, can separate in space the circuit board and input/output cables so that the circuit boards can be hot pluggable without removing cables in advance, and the efficiency of maintenance can be greatly improved. Considering the standard of IEC 60529, the level of chassis is over IP65 which is expected to prevent from dust and jetting water.

The advantages of the chassis are providing reliable and stable integrated structure, EMC shielding, heat dissipation and efficient maintenance.
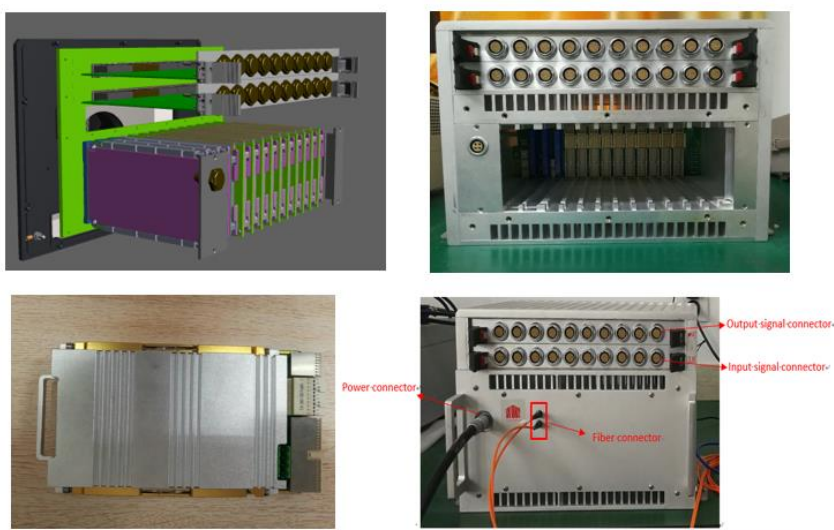

Fig.10 Design and mockup of the chassis

To increase EM-shielding performance and to enhance heat removal, metal plates were used to wrap the pre-amplifier and mid-amplifier.

The power of each card is estimated to be $11.2 \mathrm{~W}$ by summing the power of 32 chips. The heat removal of the card with air/water was simulated, setting air temperature as $30^{\circ} \mathrm{C}$. As shown in Fig.11, air cooling can keep temperature of electronics chips under $41^{\circ} \mathrm{C}$, while water cooling will have better performance.

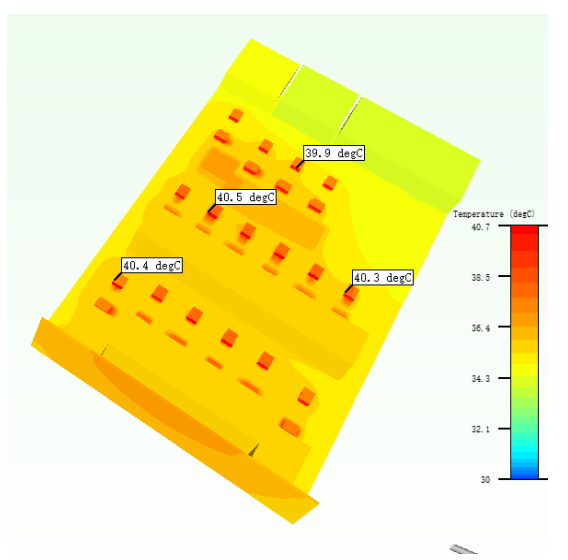

Fig.11 Result of heat dissipation simulation of the card

In addition, magnetic shielding is considered during the design of chassis. Fig.12 indicates the static magnetic field for L1 level in ITER Tokamak [8], and the stray field where the pre-amplifiers and mid-amplifiers will be located could reach respectively $120 \mathrm{mT}$ and $75 \mathrm{mT}$ during 'Full Scenario 17MA'.

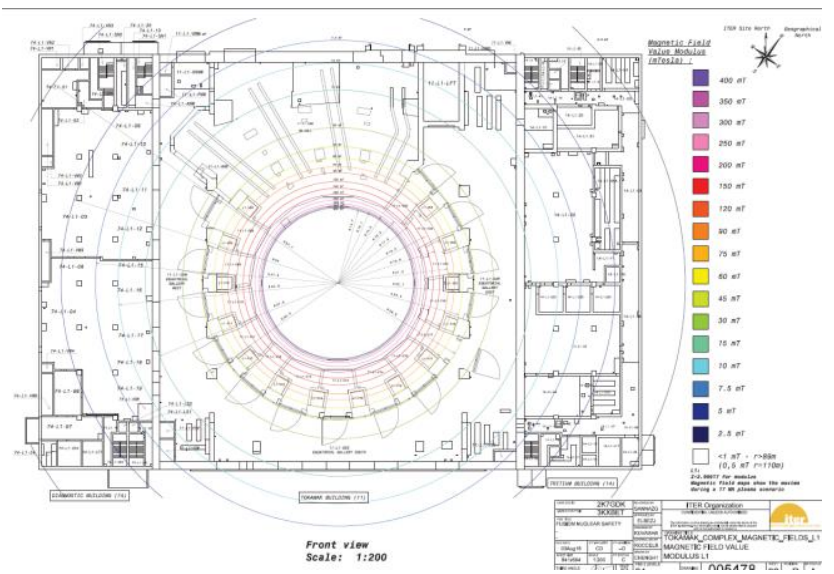

Fig.12 Magnetic radiation map of static field in ITER L1 during 17MA plasma scenario

The components used in the pre-amplifier and mid-amplifier are common components in the market, which may be not resistant to magnetic environment. As shown in Table.1 [9], the magnetic non-qualification threshold for pre-amplifier and mid-amplifier is supposed to be $5 \mathrm{mT}$. Therefore, magnetic shielding may be needed for these components. On the other hand, the electronics also need to meet the EMC requirements. As a result, customized chassis and dedicated static magnetic shielding enveloping the chassis are designed.

Table.1 EEE thresholds for magnetic field qualification

\begin{tabular}{|l|l|l|}
\hline $\begin{array}{l}\text { Static magnetic } \\
\text { field }\end{array}$ & Non critical & Critical \\
\hline Less than $5 \mathrm{mT}$ & $\begin{array}{l}\text { Design with components selected } \\
\text { for low susceptibility. }\end{array}$ & $\begin{array}{l}\text { Qualification tests* at 2 times the } \\
\text { expected induction (i.e. with a } \\
\text { margin of 6dB). }\end{array}$ \\
\hline $5 \mathrm{mT}$ to $10 \mathrm{mT}$ & $\begin{array}{l}\text { Check of bill of materials against } \\
\text { component susceptibility. } \\
\text { In case of doubt, a qualification } \\
\text { test will be requested. }\end{array}$ & $\begin{array}{l}\text { Qualification tests* at 2 times the } \\
\text { expected induction (i.e. with a } \\
\text { margin of 6dB). }\end{array}$ \\
\hline Over $10 \mathrm{mT}$ & $\begin{array}{l}\text { Qualification tests* at 2 times the } \\
\text { expected induction (i.e. with a a } \\
\text { margin of } 6 \mathrm{~dB} \text { ). }\end{array}$ & $\begin{array}{l}\text { Qualification tests* at 2 times the } \\
\text { expected induction (i.e. with a } \\
\text { margin of } 6 \mathrm{~dB} \text { ). }\end{array}$ \\
\hline
\end{tabular}


The static magnetic shielding is put outside the chassis. Permalloy will be used as shielding material which has high permeability and saturation, and its magnetic relative permeability is about $4 \times 10^{4}$ which is 10 times higher than commercial type iron. The composition of this permalloy (Model: 1J85) is shown in table.2. As shown in Fig.13, the simulation result is that $1 \mathrm{~mm}$ thickness of shielding is enough to attenuate magnetic field from $120 \mathrm{mT}$ to below $5 \mathrm{mT}$. Considering the support of the structure, it is recommended to use 1.5-2 mm thickness plates for static magnetic shielding.

Table.2 Composition of permalloy

\begin{tabular}{|l|c|c|c|c|c|c|c|c|c|}
\hline \multicolumn{10}{|c|}{ Composition-(\%) } \\
\hline Model & $\mathrm{C}$ & $\mathrm{Mn}$ & $\mathrm{Si}$ & $\mathrm{P}$ & $\mathrm{S}$ & $\mathrm{Ni}$ & $\mathrm{Mo}$ & $\mathrm{Cu}$ & $\mathrm{Fe}$ \\
\hline $1 \mathrm{~J} 85$ & $\leqslant 0.03$ & $0.3-0.6$ & $0.15-0.3$ & $\leqslant 0.02$ & $\leqslant 0.02$ & $79-81$ & $4.8-5.2$ & $\leqslant 0.2$ & $14-16$ \\
\hline
\end{tabular}

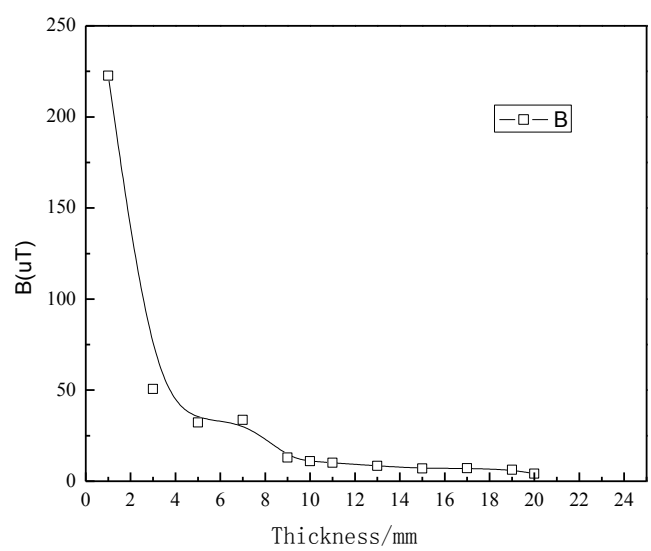

Fig.13 The static magnetic field inside the shielding box with changing thickness

\section{NEUTRON IRRADIATION TEST IN REACTOR}

The RXC camera has been tested in strong neutron radiation environment in fission reactor. The reactor power can reach $20 \mathrm{MW}$ in full power operation. The purpose of the test is mainly to know electronics performance in strong nuclear environment. The reactor power is $100 \mathrm{~kW}$ in this test, and the neutron flux is $5.84 \times 10^{9} \mathrm{n} \cdot \mathrm{cm}^{-2} \cdot \mathrm{s}^{-1}$ (1MeV equivalent) in the radiation room where the detector, pre-amplifier, mid-amplifier and cables under test received accumulated neutron fluence over $9.89 \times 10^{13} \mathrm{n} \cdot \mathrm{cm}^{-2}(1 \mathrm{MeV}$ equivalent), and the quality of output signal was monitored in real time. The total radiation time is $16928 \mathrm{~s}$ and the ratio of $\mathrm{n} / \gamma$ is $7.7 \times 10^{9} \mathrm{n} \cdot \mathrm{cm}^{-2} \cdot \mathrm{rad}^{-1}$.

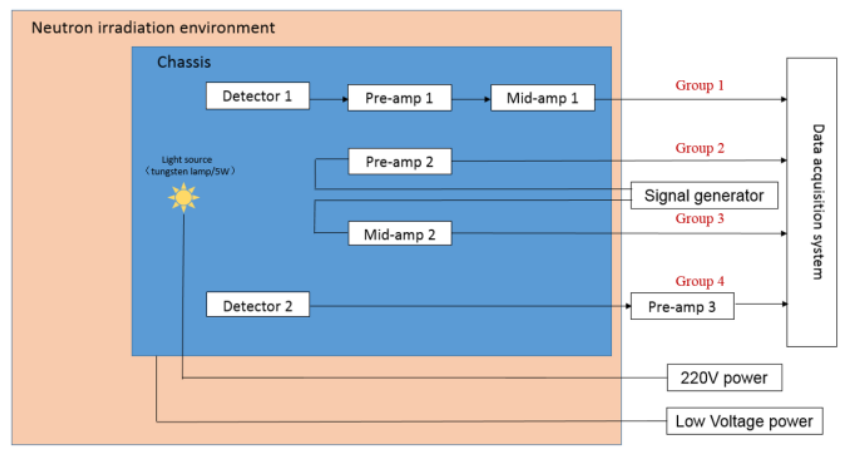

Fig.14 Layout sketch of electronics neutron irradiation test in the

\section{reactor}

As shown in Fig.14, four groups of detector, pre-amplifier and mid-amplifier are exposed in irradiation environment. In groups 2-4, only pre-amplifier, mid-amplifier and detector is put inside the irradiation room, respectively; in group 1, the detector, pre-amplifier and mid-amplifier are both put inside the irradiation room. It should be noted that, in group 2-3, the input signal comes from signal generator; in group 1 and 4 , visible light source (tungsten lamp) is used to illuminate detectors. This experiment arrangement can identify in real time whether the detector, pre-amplifier or mid-amplifier is impaired. To verify the influence of FPGA code on mid-amplifier SEE, the odd channels and even channels of the two amplifier cards are written with different codes. The odd channels are configured only once in the code on receiving command from I\&C, while the even channels are automatically configured several times per second on receiving command.

As shown in Fig.15 and Fig.16, a customized PXIE chassis is designed for neutron irradiation test in fission reactor, mainly for support and power supply of tested components. The tungsten lamp and detectors are in left side and right side of the chassis panel respectively. Two pre-amplifier and two detectors are in the same vertical plane where the neutron flux is the same.

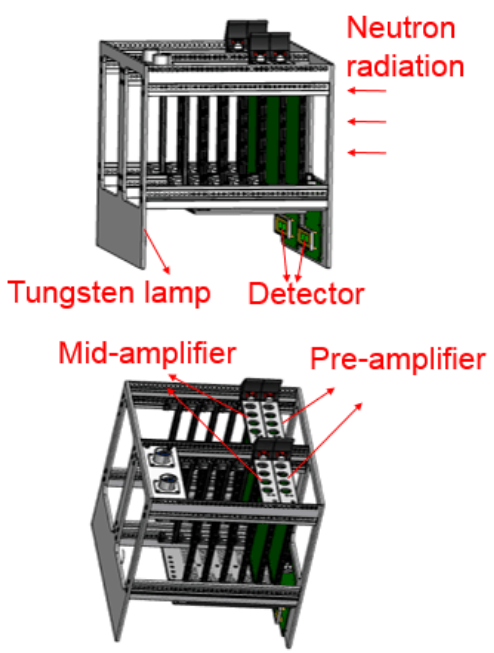

Fig.15 3D model of chassis for neutron irradiation test in reactor 


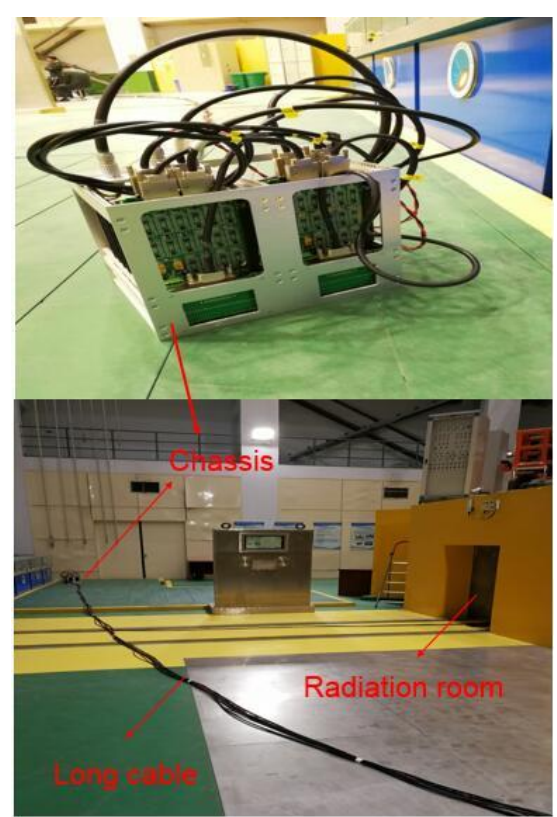

Fig.16 Photo of chassis and the radiation room

As shown in Fig.17, DAQ system put outside the radiation region is used to record and display the output of pre-amplifier and mid-amplifier in real time. The change of signal amplitude is used to assess damage level due to neutron irradiation.

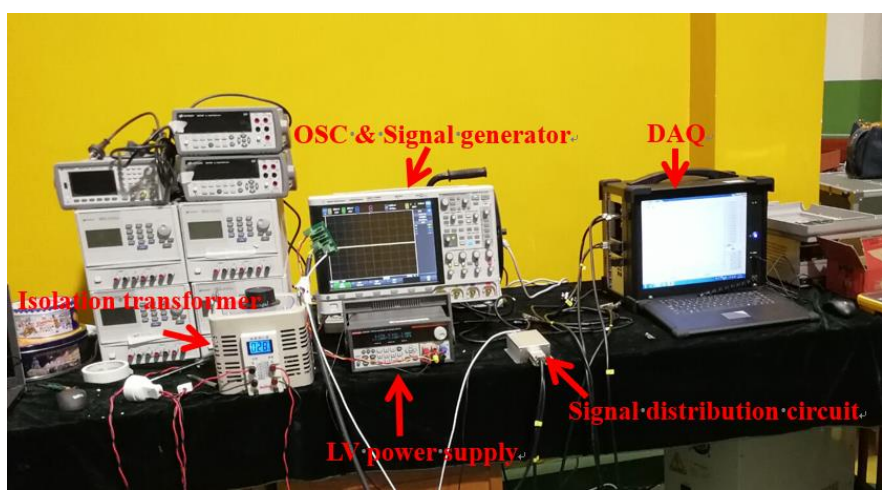

Fig.17 Photo of DAQ system and low voltage power supply

The test results are shown in Fig.18, Fig.19, Fig.20 and Fig.21. For group 2, pre-amplifier works well in the whole test and the amplification of pre-amplifier increased $0.83 \%$. For group 1, only 1 SEE was found in one of the even channel of mid-amplifier. For group 3, 5 SEEs were found in the mid-amplifier which can be recovered by restarting, among which $1 \mathrm{SEE}$ was found in all the channels and these channels saturated, and other SEEs were found only in even channels. When neutron fluence reached $6.4 \times 10^{13} \mathrm{n} / \mathrm{cm}^{2}$, mid-amplifiers' output began to decrease. By testing after experiment, there were two power supply chips in mid-amplifier damaged (note that no radiation resistant power chip was used in mid-amplifier). For group 4, the detector is not fully damaged, but the responsivity to visible light decreased $40 \%$.

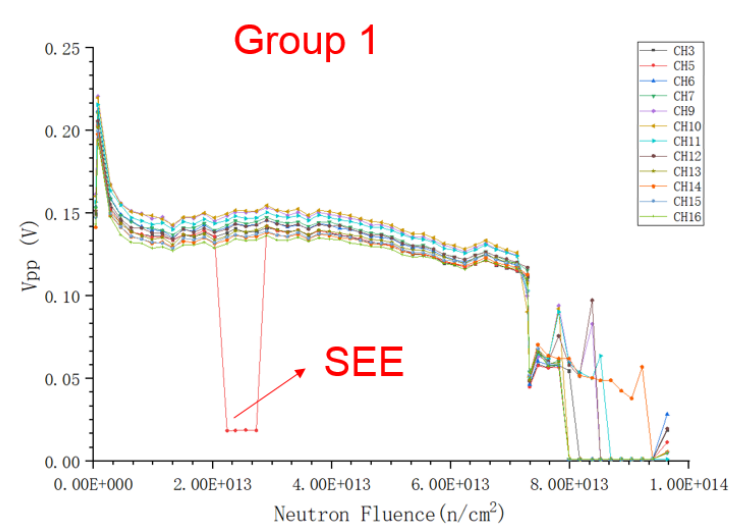

Fig.18 The result of group 1

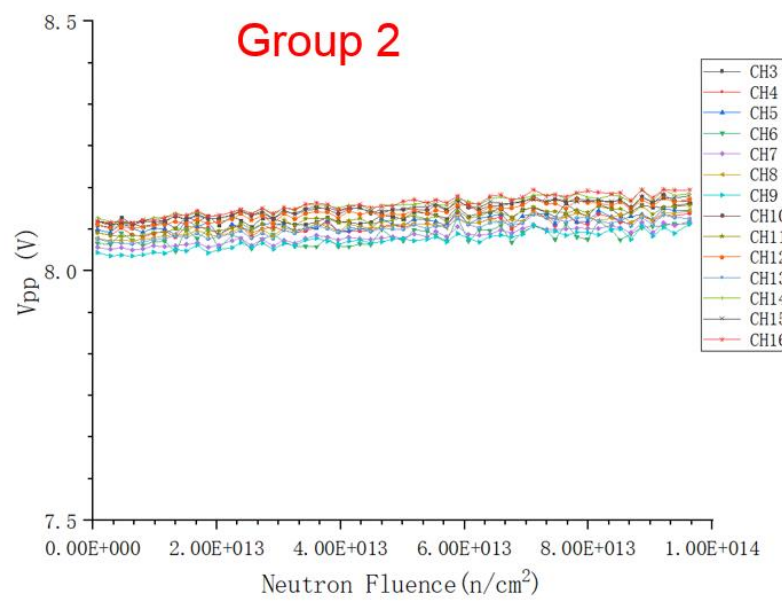

Fig.19 The result of group 2

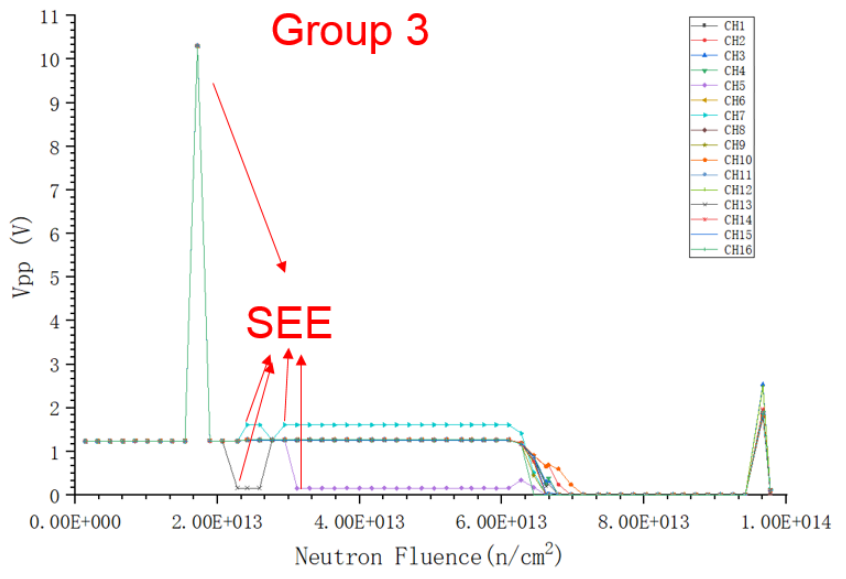

Fig.20 The result of group 3 


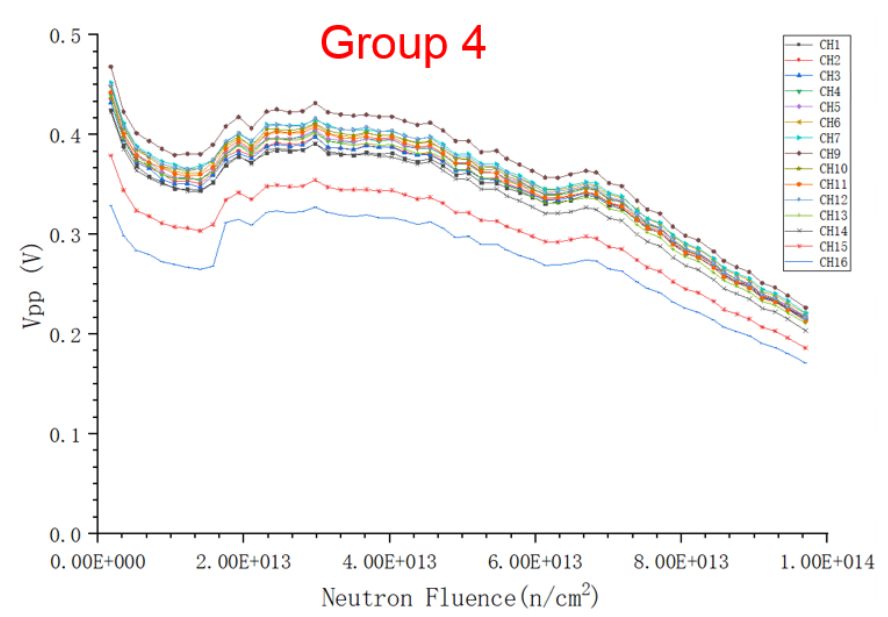

Fig.21 The result of group 4

\section{RXC SYSTEM TEST IN EAST TOKAMAK}

The diode array detector, pre-amplifier, DAQ system, customized chassis and radiation-hard cables to be used in ITER RXC have been tested in Experimental Advanced Superconducting Tokamak (EAST) experiments.

In the tests, the detector available is Centronic LD35(24)-5T which contains 35 elements each with active area of $12 \mathrm{~mm} \times 2 \mathrm{~mm}$ as shown in Fig. 22 .

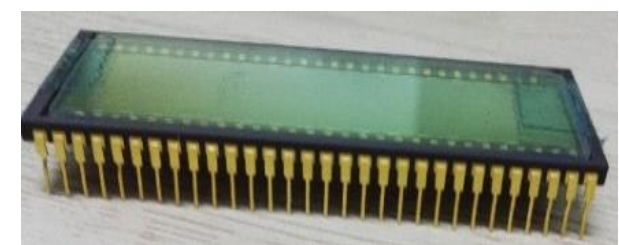

Fig.22 Picture of detector

The 0.5 meter Kapton cable is used from detector to feedthrough, 25 meter HELUKABEL $(18 \times 2 \times 0.25)$ cable is used from feedthrough to pre-amplifier, and 100 meter HELUKABEL $(18 \times 2 \times 0.25)$ cable is used from pre-amplifier to DAQ as shown in Fig. 23.

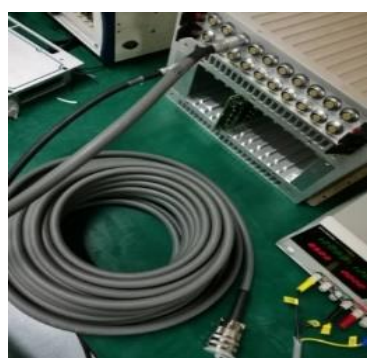

Fig.23 Picture of HELUKABEL $(18 \times 2 \times 0.25)$ cable

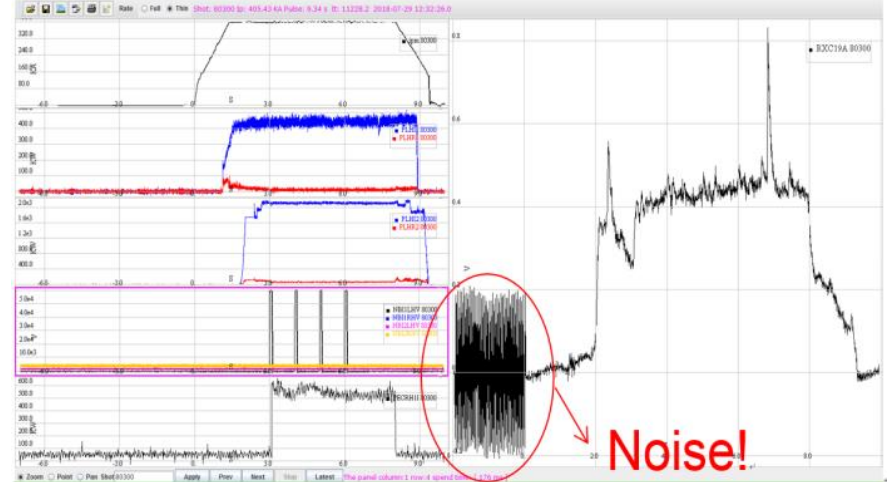

Fig.24 Picture of discharge signals from webscope in EAST

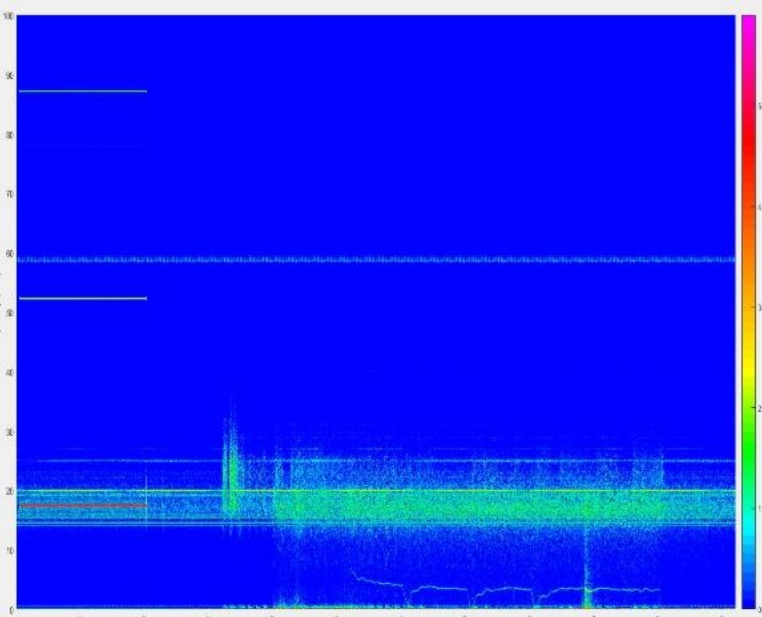

Fig.25 Frequency spectrum with time of the $\mathrm{x}$-ray signal

As shown in Fig.24 and Fig.25, during the plasma discharge, as the ECRH, NBI and lower hybrid waves (LHWs) were jointly injected to the plasma, the whole electronics system worked well. However, there was a lot of noise found in soft $\mathrm{x}$-ray signal, especially before the discharge was started.

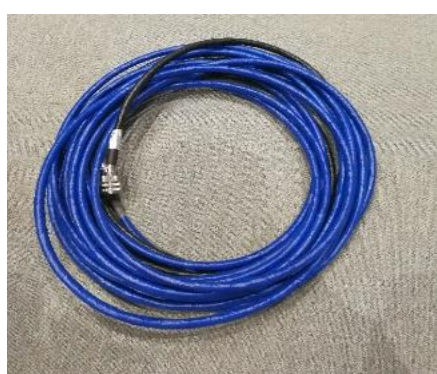

Fig.26 Picture of HELUKABEL $(18 \times 2 \times 0.25)$ cable with aluminum foil shielding added 


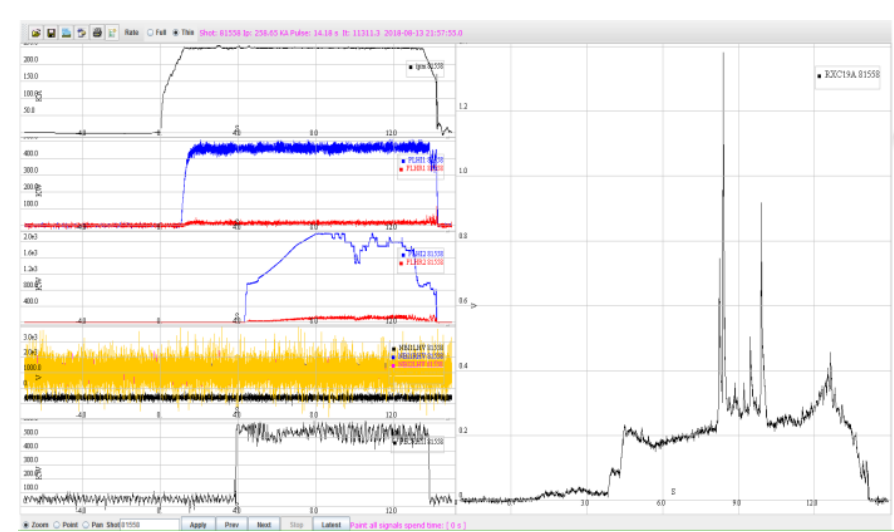

Fig.27 Picture of discharge signals from webscope in EAST

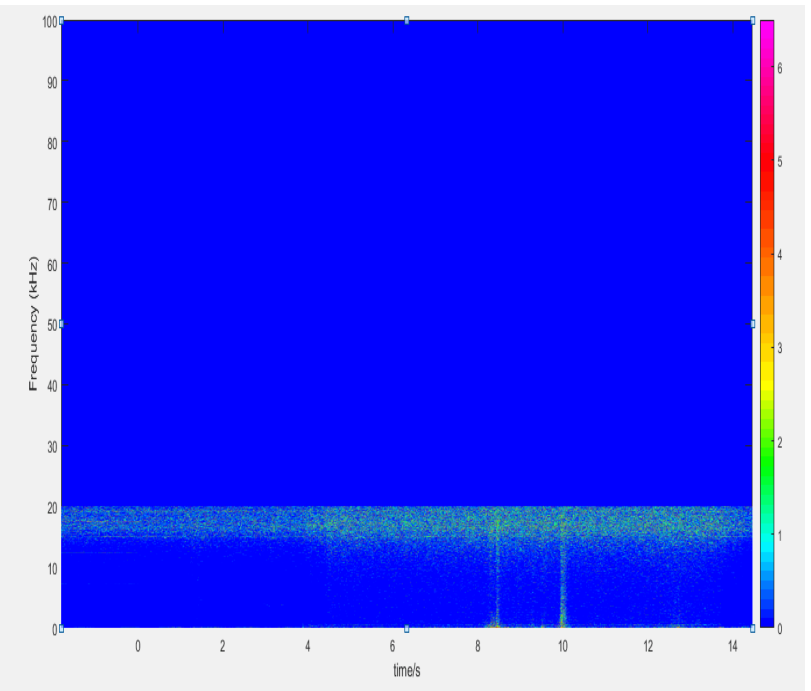

Fig.28 Frequency spectrum with time of the x-ray signal after using $\mathrm{Al}$ shielding for cables

As shown in Fig.26, Fig.27 and Fig.28, as aluminum foil shielding was added to the cable, the signal became well immediately (seen from Fig.27 and 28). This indicates the signal quality can be improved by adding aluminium shielding.

\section{CONCLUSION}

In final design phase of ITER Radial X-ray Camera, enhancement of irradiation resistance research of compact electronics has been done. Power chip and FPGA in the circuit which are identified as weak parts have been improved by radiation resistance design. Considering influence of nuclear environment on worker exposure to radiation and optimization of space reservation limited in port cell to allow for more space to put nuclear shielding, easiness of maintenance, compact electronics structure and remote state monitoring are designed, while magnetic shielding was also considered. To exploit and verify the radiation performance, the electronics are tested in irradiation experiment in reactor. No problem was found in pre-amplifier with radiation resistant power chip; the responsivity of detectors decreased $40 \%$; the mid-amplifier with optimized FPGA code was found less SEEs than that in previous experiment. The electronics were also integrated and tested in EAST tokamak, and found work well in EAST experiments with various ancillary heating.

\section{ACKNOWLEDGMENT}

ITER is a Nuclear Facility INB-174. The views and opinions expressed herein do not necessarily reflect those of the ITER Organization. Thanks to people in ITER Organization and other organizations or institutes supporting RXC design. Special thanks to Robin Barnsley, Antoine Sirinelli, Artur Malaquias, Barry Alper, and Michael Walsh.

This work was supported by the National Nature Science Foundation of China under Grant NO.11605240 and NO.11605243.

\section{REFERENCES}

[1] L. Hu, et al., "Preliminary design and R\&D of ITER diagnostic-radial X-ray camera", Nuclear Inst. and Methods in Physics Research A, vol. 870, pp. 50-54, Jul. 2017.

[2] L. Hu, et al., "Design and Test of Irradiation-Related components in ITER Radial X-Ray Camera", IEEE TRANSACTIONS ON NUCLEAR SCIENCE, vol.65, No.9, pp. 2398-2403, Sep. 2018.

[3] http://www.ti.com/product/OPA2140/technicaldocuments

[4] http://www.ti.com/product/THS4131/technicaldocuments

[5] https://www.st.com/en/space-products/rhfl4913a.html

[6] https://www.st.com/en/space-products/rhfl7913a.html

[7] https://www.intel.co.uk/content/www/uk/en/products/programmable/fpga /max-10.html

[8] R. Roccella, private communication, Oct 2018.

[9] D. Hamilton, private communication, May 2012. 